\title{
Correlations of post-implant regional dosimetric parameters at 24 hours and one month, with clinical results of low-dose-rate brachytherapy for localized prostate cancer
}

\author{
Eiichiro Okazaki, MD, PhD', Katsuyuki Kuratsukuri, MD, PhD², Kentaro Ishii, MD, PhD, Tomoaki Tanaka, MD, PhD² \\ Ryo Ogino, MD, PhD , Tomohiro Nishikawa, MD5. Hideyuki Morimoto, MD5, Masako Hosono, MD, PhD ${ }^{5}$, \\ Yukio Miki, MD, $\mathrm{PhD}^{5}$ \\ 'Department of Radiation Oncology, Saiseikai Nakatsu Hospital, ${ }^{2}$ Department of Urology, Osaka City University Graduate School \\ of Medicine, ${ }^{3}$ Department of Radiation Oncology, Tane General Hospital, ${ }^{4}$ Department of Radiation Oncology. Osaka Minami Medical \\ Center, ${ }^{5}$ Department of Diagnostic and Interventional Radiology/Radiation Oncology, Osaka City University Graduate School of Medicine, \\ Osaka, Japan
}

\begin{abstract}
Purpose: To evaluate the correlations of post-implant regional dosimetrics at 24 hours $(24 \mathrm{~h})$ and 1 month after implant procedures, with clinical outcomes of low-dose-rate (LDR) brachytherapy for localized prostate cancer.

Material and methods: Between January 2008 and December 2014, 130 consecutive patients treated for localized prostate cancer, receiving definitive iodine-125 ( $\left.{ }^{125} \mathrm{I}\right)$ brachytherapy treatment were retrospectively analyzed. All patients underwent post-implant CT imaging for dosimetric analysis at $24 \mathrm{~h}$ and 1 month after implantation procedure. Prostate contours were divided into quadrants: anterior-superior (ASQ), posterior-superior (PSQ), anterior-inferior (AIQ), and posterior-inferior (PIQ). Predictive factors and cut-off values of biochemical failure-free survival (BFFS) and toxicities of LDR brachytherapy were analyzed.

Results: The median follow-up time was 69.5 months. Seven patients $(5.4 \%)$ had biochemical failure. The 3-year and 5-year BFFS rates were $96.7 \%$ and $93.1 \%$, respectively. On multivariate analysis, prostate-specific antigen and Gleason score were significant prognostic factors for biochemical failure. $\mathrm{D}_{90}$ (the minimal dose received by $90 \%$ of the volume) of PSQ and PIQ at $24 \mathrm{~h}$, and $\mathrm{D}_{90}$ of PSQ at 1 month were also significant factors. The cut-off values of PSQ $\mathrm{D}_{90}$ were $145 \mathrm{~Gy}$ at $24 \mathrm{~h}$ and $160 \mathrm{~Gy}$ at 1 month. $\mathrm{D}_{90}$ of the whole prostate was not significant at $24 \mathrm{~h}$ and at 1 month. $\mathrm{D}_{90}$ of PSQ at 1 month was a significant factor for rectal hemorrhage.

Conclusions: Post-implant $\mathrm{D}_{90}$ of PSQ is significantly associated with BFFS for localized prostate cancer not only at 1 month, but also at 24 hours. $D_{90}$ of PSQ at 1 month is also a significant factor for rectal hemorrhage.

Key words: brachytherapy, low-dose-rate, prostate cancer, seeds.

\section{Purpose}

Prostate cancer is one of the most common malignancies in men and a major cause of cancer death [1]. Prostate-specific antigen (PSA) screening enables the detection of early and localized prostate cancer. There are many therapeutic options for patients with localized prostate cancer, including androgen deprivation therapy (ADT), radical prostatectomy, external beam radiation therapy, and brachytherapy. Permanent seed brachytherapy, also known as low-doserate (LDR) brachytherapy, allows for a higher dose of ra- diation to the prostate with a steep dose gradient to surrounding normal tissues. Previous reviews have shown that outcomes with brachytherapy for localized prostate cancer are comparable to those with other therapeutic modalities, including radical prostatectomy and external beam radiation therapy $[2,3,4]$. Furthermore, brachytherapy is less invasive than surgery and can be performed in a shorter period than external beam radiation therapy.

The implant quality of LDR brachytherapy can be assessed by performing a dosimetric evaluation based on post-implant computed tomography (CT) and/or magnetic 
resonance imaging (MRI) [5,6], and dosimetric parameters such as D90 (the minimum dose received by $90 \%$ of the volume) and V100 (the percentage of the volume receiving $100 \%$ of prescribed dose) of the whole prostate are mainly used for analysis [5]. Several reports have shown that these dosimetric parameters are significant factors in predicting biochemical failure $[7,8,9,10,11]$, although there is a controversy about the usefulness of the dosimetric parameters in predicting biochemical failure [12,13,14]. Many of these reports have mentioned dosimetric parameters of the whole prostate, and there is a small number of reports evaluating dose distribution in different regions of the prostate $[15,16,17,18,19]$. It is known that there are dose deviations from prescribed dose in different regions of the prostate; in particular, the anterior and basal regions tend to be underdosed compared to other regions $[17,20]$. There are few reports assessing the relationships between regional dose distribution of the prostate and therapeutic outcomes, including biochemical failure and radiation toxicities $[19,20]$.

The optimal timing for obtaining post-implant CT and/or MRI also remains a debated issue. Prostatic edema arises in the first $24 \mathrm{~h}$ after implantation, and post-implant imaging obtained within $24 \mathrm{~h}$ results in lower calculated doses [6,21]. Some studies suggest an interval of 2 to 6 weeks after implantation for the effect of prostatic edema to decrease [22,23], while other reports recommends dosimetric evaluation within $24 \mathrm{~h}$, because this allows for immediate correction of a dose deficiency, if necessary $[24,25,26]$. To the best of our knowledge, there have been no reports about the correlations between therapeutic outcomes and regional dosimetry of the prostate using images obtained at $24 \mathrm{~h}$ after the procedure.

The purposes of this study were to evaluate the correlations between post-implant regional dosimetrics and the outcomes of LDR brachytherapy, and to assess the effectiveness of post-implant CT obtained early, at 24 hours, compared with that obtained later, at 1 month after implantation.

\section{Material and methods \\ Patients' characteristics}

This study was approved by institutional review board, and was conducted in accordance with the principles of the Declaration of Helsinki. From January 2008 to December 2014, outcome data of consecutive patients treated with LDR brachytherapy for localized prostate cancer at Osaka City University Hospital were retrospectively analyzed. Patients were staged according to the guidelines of the American Joint Committee on Cancer, and they were classified into prognostic risk groups according to the National Comprehensive Cancer Network (NCCN: www. nccn.org) guidelines. Low- and intermediate-risk patients who received LDR brachytherapy as monotherapy were included in this study. Patients treated with combined external beam radiation therapy or those who missed the post-implant CT examination were excluded. Neoadjuvant ADT was generally administered to patients with prostate volumes $>40 \mathrm{cc}$ to reduce the volumes.

\section{Pre-implant planning}

MRI-based pre-implant volume studies were performed with a patient in supine position using 1.5T Avanto scanner (Siemens Medical Solutions, Erlangen, Germany) at a median of 7 weeks (range, 2-42 weeks) before implantation. T2-weighted 3D MR images of $2.5 \mathrm{~mm}$ thickness were imported into the treatment planning system (TPS). Through March 2014, an Interplant (version 3.4.0, Computerized Medical Systems, Champaign, IL, USA) TPS was used for pre-implant and real-time planning, and from April 2014, an Oncentra (version 4.2.2, Nucletron, Veenendaal, The Netherlands) TPS was used. The dosimetry of pre-implant planning aimed for a prostate $\mathrm{V} 100$ of $>95 \%$, a prostate D 90 of $>110 \%$ and $<130 \%$ of the prescribed dose, a prostate V150 (the volume receiving $150 \%$ of the prescribed dose) of $<60 \%$, a urethral D30 (the minimum dose received by $30 \%$ of the volume) of $<150 \%$, and a rectal V100 of $<0.2$ cc.

\section{Implant procedure}

The implant procedure was performed under spinal anesthesia in dorsal lithotomy position. For all patients, iodine-125-free seeds (BARD, BrachySource model, Covington, GA, USA) with an apparent activity of $0.342 \mathrm{mCi}$ and an air kerma strength of $0.432 \mathrm{U}\left(\mu \mathrm{Gym}^{2} \mathrm{~h}^{-1}\right)$ were used, and the prescribed brachytherapy dose was $145 \mathrm{~Gy}$. The seeds were implanted transperineally with a Mick applicator (Mick Radio-Nuclear Instruments, New York, NY, USA) using real-time transrectal ultrasonography. A modified peripheral loading technique was used to deliver the dose to the prostate, avoiding placement of seeds close to the urethra [27]. Dose-volume constraints for the prostate, urethra, and rectum were the same as the pre-implant planning.

\section{Post-implant dosimetry}

Post-implant axial CT images of the pelvis with the patient in supine position using an Asteion (Toshiba Medical Systems, Tokyo, Japan) at $2.0 \mathrm{~mm}$ thickness were taken $24 \mathrm{~h}$ and 1 month (median, 34 days) after the implant procedure. Post-implant dosimetry was re-analyzed with Oncentra TPS, including what was previously calculated with Interplant (before April 2014) by a single radiation oncologist (EO). To mitigate the influence of metal artifacts of the seeds and variation of contouring related practitioner on CT images, CT-MRI image fusion for delineation of volumes was done for all patients on Oncentra TPS, using MRI images obtained at pre-implant volume studies. The prostate volume was divided into four quadrants: anterior-superior quadrant (ASQ), posterior-superior quadrant (PSQ), anterior-inferior quadrant (AIQ), and posterior-inferior quadrant (PIQ). The superior-inferior and anterior-posterior segments were divided by the midpoint of the prostate on reconstructed images (Figure 1).

\section{Patient follow-up}

The date of implantation was considered day 0 for the analysis of follow-up duration. Patients were assessed every 2 or 3 months during the first to third year, and every 

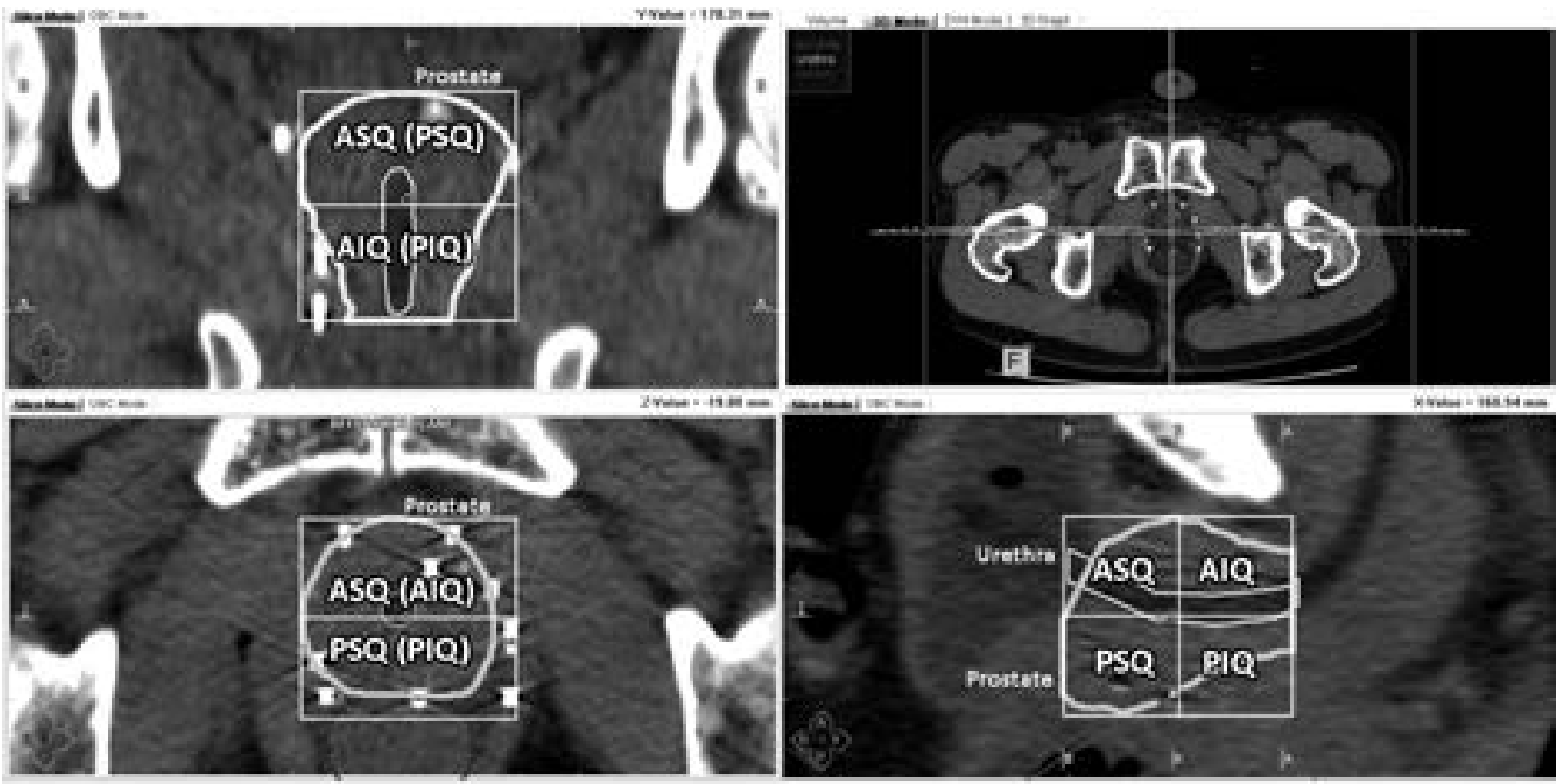

Fig. 1. Separation of the prostate into four quadrants

$A S Q$ - anterior-superior quadrant, PSQ - posterior-superior quadrant, AIQ - anterior-inferior quadrant, PIQ - posterior-inferior quadrant

3 to 6 months at 3-10 years after brachytherapy. During clinical follow-up visits, serum PSA levels and toxicity data were collected. Biochemical failure was determined according to the Phoenix definition [28]. Genitourinary (GU) and gastrointestinal (GI) toxicities were documented using the Common Terminology Criteria for Adverse Events version 4.0. Acute toxicity was defined as that occurring within the first 12 months after implantation, and late toxicity was defined as that developing after 12 months.

\section{Statistical analyses}

Statistical analysis was performed to compare continuous variables using paired or unpaired $t$-tests and oneway ANOVA with Tukey's honest significant difference (HSD) for post-hoc testing. Univariate and multivariate analyses were performed using Cox proportional hazards models with corresponding 95\% confidence intervals. Variables with a $p$ value $<0.2$ on univariate analyses were incorporated into multivariate analysis. To avoid multicollinearity, univariate and multivariate analyses were performed separately by the post-implant dosimetric parameters at $24 \mathrm{~h}$ or 1 month. Coordinate points of a receiver operating characteristic (ROC) curve were used to identify significant dosimetric parameters for predicting biochemical failure and toxicities. Survival curves were estimated using Kaplan-Meier method to determine biochemical failure-free survival (BFFS), and the log-rank test was used to evaluate differences between groups. Statistical analyses were performed using $\mathrm{R}$ environment (version 3.2.2) available from http:/ / www.R-project.org.

\section{Results}

From January 2008 to December 2014, 132 consecutive patients with low- and intermediate-risk prostate cancer underwent LDR brachytherapy as monotherapy at our institution. Two patients were excluded for not undergoing post-implant CT, and a total of 130 patients were eligible for this study. Median age was 69 years (range, 42-82 years), and median follow-up time was 69.5 months (range, 10.6-112.9 months). Patients' characteristics are listed in Table 1 . Sixty patients received neoadjuvant ADT approximately 3-6 months before brachytherapy (34 patients with luteinizing hormone-releasing hormone agonist, 18 with maximum androgen blockade, and 8 with anti-androgen). No deaths occurred during the follow-up period. Seven patients $(5.4 \%)$ had biochemical failure. The 3-year and 5-year BFFS rates for all patients were $96.7 \%$ and $93.1 \%$, respectively. The 5-year BFFS rates for low- and intermediate-risk patients were $97.5 \%$ and $88.5 \%$, respectively ( $p=0.087$ with log-rank test).

\section{Dosimetric analysis}

Post-implant dosimetric data are shown in Table 2A and $2 \mathrm{~B}$. The prostate D90 at 1 month was significantly higher than that at $24 \mathrm{~h}$ for all quadrants $(p<0.001$ for all quadrants). D90 of each quadrant at $24 \mathrm{~h}$ were strongly correlated with those at 1month $(r=0.63-0.71$, and $p<0.001$ for all with Pearson's correlation). With multiple comparisons of dosimetric parameters for each quadrant, D90 of PIQ was significantly lower than that of the other quadrants at $24 \mathrm{~h}(p<0.001$ with Tukey HSD). At 1 month, D90 of ASQ was significantly lower than that of the other quadrants, and D90 of AIQ was significantly higher than that of the others $(p<0.001)$.

\section{Dosimetry and therapeutic outcomes}

The results of univariate and multivariate analyses of prognostic factors for predicting biochemical failure at $24 \mathrm{~h}$ and 1 month are shown in Table 3A and 3B, respec- 
Table 1. Patients' characteristics

\begin{tabular}{|c|c|}
\hline Variable & $n(\%)$ \\
\hline \multicolumn{2}{|l|}{ Age (years) } \\
\hline Median & 69 \\
\hline Range & $42-82$ \\
\hline \multicolumn{2}{|c|}{ Pretreatment PSA (ng/ml) } \\
\hline Median & 6.6 \\
\hline Range & $3.6-15.1$ \\
\hline \multicolumn{2}{|l|}{ Gleason score } \\
\hline$\leq 6$ & $97(74.6)$ \\
\hline 7 & $33(25.4)$ \\
\hline \multicolumn{2}{|l|}{ Clinical T stage } \\
\hline T1c & $64(49.2)$ \\
\hline $\mathrm{T} 2 \mathrm{a}$ & $48(36.9)$ \\
\hline $\mathrm{T} 2 \mathrm{~b}$ & $8(6.2)$ \\
\hline $\mathrm{T} 2 \mathrm{c}$ & $10(7.7)$ \\
\hline \multicolumn{2}{|c|}{ NCCN risk group } \\
\hline Low & $68(52.3)$ \\
\hline Intermediate & $62(47.7)$ \\
\hline \multicolumn{2}{|c|}{ Neoadjuvant ADT } \\
\hline No & $70(53.8)$ \\
\hline Yes & $60(46.2)$ \\
\hline \multicolumn{2}{|c|}{ Pre-implant MRI prostate volume (cc) } \\
\hline Median & 23.2 \\
\hline Range & $8.0-68.7$ \\
\hline \multicolumn{2}{|c|}{ Number of seeds } \\
\hline Median & 67 \\
\hline Range & $43-100$ \\
\hline \multicolumn{2}{|c|}{ Follow-up (months) } \\
\hline Median & 69.5 \\
\hline Range & $10.6-112.9$ \\
\hline
\end{tabular}

PSA - prostate specific antigen, NCCN - National Comprehensive Cancer Network, $A D T$ - androgen deprivation therapy, MRI - magnetic resonance imaging

tively. On multivariate analysis, PSA and Gleason score were significant prognostic factors for biochemical failure, while T stage and risk group were not significant, both at 24 hours and at 1 month. For dosimetric parameters, D90 of PSQ (PSQ D90 24h ), PIQ at 24 hours, and D90 of PSQ at 1 month (PSQ D90 $1 \mathrm{~m}$ ) were significant factors on multivariate analysis $(p=0.002,0.048$, and 0.014 , respectively), while D90 of the whole prostate was not significant $(p=$ 0.15 at $24 \mathrm{~h}$ and 0.77 at 1 month, respectively). D90 of PSQ was a significant prognostic factor in both analyses at
24 hours and 1 month. Therefore, a ROC analysis to identify the best cut-off value of PSQ D90 was performed. The cut-off values of PSQ D90 were 101.7\% (area under the curve $[\mathrm{AUC}]=0.80$, sensitivity $=100 \%$, specificity $=$ $72.4 \%$ ) at 24 hours, and $112.3 \%$ (AUC $=0.74$, sensitivity $=85.7 \%$, specificity $=65.9 \%$ ) at 1 month. These cut-off values of PSQ D90 was simplified as 145 Gy $(100.0 \%)$ at $24 \mathrm{~h}$, and 160 Gy $(110.3 \%)$ at 1 month. Kaplan-Meier Curves with these cut-off values of PSQ D90 are shown in Figures $2 \mathrm{a}$ and $2 \mathrm{~b}$, and the log-rank tests of these curves were significant (145 Gy at $24 \mathrm{~h}: p=0.033 ; 160 \mathrm{~Gy}$ at 1 month: $p=0.037)$.

No grade 3 acute and chronic GU toxicity was observed. Twenty-nine patients $(22.3 \%)$ experienced late grade 2 GU toxicities (mainly urinary urgency and retention). During the follow-up period, 20 patients $(15.4 \%)$ experienced at least one episode of hematuria (median, 36.6 months; range, 2.6-75.4 months). There was no significant relationship between an episode of hematuria and post-implant dosimetric parameters. No grade 3 acute GI toxicity was observed. Eight patients $(6.0 \%)$ experienced rectal hemorrhage (median, 17.1 months; range, 10.3-38.7 months). One patient received argon plasma coagulation. Comparing dosimetric parameters between patients who experienced rectal hemorrhage and others, there were significant differences in PSQ D90 $1 \mathrm{~m}$ (137.1\% [198.8 Gy] vs. $117.8 \%$ [170.8 Gy], $p=0.037)$, and V100 of the rectum at 24 hours ( $0.64 \mathrm{cc}$ vs. $0.31 \mathrm{cc}, p=0.038)$ and at 1 month ( 1.51 cc vs. 0.83 cc, $p=0.008$ ) with the $t$-test. Cut-off values of each parameter on ROC curve analysis were $134.4 \%$ $(194.9$ Gy, $\mathrm{AUC}=0.69$, sensitivity $=71.4 \%$, specificity $=$ $76.2 \%)$ for PSQ D90 $0_{1 \mathrm{~m}}, 0.88 \mathrm{cc}(\mathrm{AUC}=0.64$, sensitivity $=$ $50.0 \%$, specificity $=90.2 \%$ ) for rectal V100 at $24 \mathrm{~h}$, and 1.36 cc $($ AUC $=0.75$, sensitivity $=71.4 \%$, specificity $=80.3 \%)$ for rectal V100 at 1 month. Kaplan-Meier curve for the cumulative risk of rectal hemorrhage with a simplified cut-off value of PSQ D90 $1 \mathrm{~m}$ (195 Gy) and rectal V100 at 1 month are shown in Figures $3 a$ and $3 b(p=0.016$ and 0.048 with the log-rank test, respectively). Unlike PSQ D90 ${ }_{1 \mathrm{~m}}$, PSQ D90 $24 \mathrm{~h}$ was not a significant factor for rectal hemorrhage $(p=0.33)$.

\section{Discussion}

The results of this study indicate that there is a significant relationship between D90 of PSQ and BFFS, and this correlation is significant not only at 1 month after the implant procedure, but also at $24 \mathrm{~h}$. D90 of PSQ is also correlated with rectal hemorrhage as a late complication of brachytherapy.

In this study, D90 of PSQ was found to be a significant predictive factor of BFFS, and D90 of the whole prostate was not significant at $24 \mathrm{~h}$ and 1 month. In a cohort of consecutive patients, Spadinger et al. $[19,20]$ confirmed that the dosimetrics of the whole prostate did not predict BFFS, and that dosimetrics of AIQ were predictors on multivariate analysis. The difference by prostatic region, PSQ, or AIQ can be affected by the different methods of prostate separation. The process of dividing the superior and inferior segments was the same in the present study, but the processes of dividing the anterior and posterior 
Table 2A. Post-implant dosimetric data for the prostate, urethra, and rectum ( $24 \mathrm{~h}$ and 1 month)

\begin{tabular}{llcccccccc} 
& & \multicolumn{4}{c}{ Prostate } & \multicolumn{2}{c}{ Urethra } & \multicolumn{2}{c}{ Rectum } \\
\cline { 2 - 10 } & & Volume $(\mathrm{cc})$ & $\mathrm{D}_{90}(\%)$ & $\mathrm{V}_{100}(\%)$ & $\mathrm{V}_{150}(\%)$ & $\mathrm{D}_{30}(\%)$ & $\mathrm{V}_{150}(\%)$ & $\mathrm{V}_{100}(\mathrm{cc})$ \\
\hline \multirow{2}{*}{$24 \mathrm{~h}$} & Median & 28.4 & 99.1 & 89.3 & 44.7 & 124.5 & 0 & 0.18 \\
\cline { 2 - 10 } & Range & $11.9-51.7$ & $52.9-125.2$ & $62-98.8$ & $19.8-76.8$ & $93.4-191.3$ & $0-75.43$ & $0-0.24$ \\
\hline \multirow{2}{*}{1 month } & Median & 22.1 & 112.6 & 94.6 & 67.4 & 159.0 & 43.9 & 0.75 \\
\cline { 2 - 10 } & Range & $9.6-46.7$ & $66.4-163.2$ & $74.0-100$ & $29.8-99.8$ & $113.2-250$ & $0-81.5$ & $0-3.5$
\end{tabular}

$D_{90}$ - minimal dose received by $90 \%$ of volume, $V_{100}$-volume receiving $100 \%$ of prescribed dose, $V_{150}$-volume receiving $150 \%$ of prescribed dose, $D_{30}-$ minimal dose received by $30 \%$ of volume

Table 2B. Post-implant dosimetric data for the quadrants of prostate ( $24 \mathrm{~h}$ and 1 month)

\begin{tabular}{|c|c|c|c|c|c|}
\hline & & \multicolumn{4}{|c|}{ Prostate } \\
\hline & & ASQ D 90 (\%) & PSQ D 90 (\%) & AIQ $D_{90}(\%)$ & PIQ D 90 (\%) \\
\hline \multirow[t]{2}{*}{$24 \mathrm{~h}$} & Median & 100.1 & 111.4 & 106.7 & 92.7 \\
\hline & Range & 54.3-191.7 & $50.4-145.3$ & $39.3-165.4$ & 38.9-147.5 \\
\hline \multirow[t]{2}{*}{1 month } & Median & 107.4 & 118.5 & 133.4 & 120.2 \\
\hline & Range & 50.4-181.8 & $56.6-184.7$ & 72.4-195.7 & 61.4-186.6 \\
\hline
\end{tabular}

$D_{90}$ - minimal dose received by $90 \%$ of volume, $A S Q$ - anterior-superior quadrant, $P S Q$ - posterior-superior quadrant, AIQ - anterior-inferior quadrant, $P I Q$ - posterior-inferior quadrant

Table 3A. Univariate and multivariate analyses of prognostic factors using variables obtained from postimplant CT at $24 \mathrm{~h}$

\begin{tabular}{|c|c|c|c|c|c|c|}
\hline \multirow[t]{2}{*}{ Variables } & \multicolumn{3}{|c|}{ Univariate analysis } & \multicolumn{3}{|c|}{ Multivariate analysis } \\
\hline & HR & $95 \% \mathrm{Cl}$ & $p$-value & $\mathrm{HR}$ & $95 \% \mathrm{Cl}$ & $p$-value \\
\hline Age & 0.977 & $0.881-1.084$ & 0.67 & & & \\
\hline PSA & 1.287 & $1.015-1.633$ & 0.037 & 1.638 & $1.159-2.315$ & 0.005 \\
\hline Gleason score ( $\leq 6$ vs. 7$)$ & 4.492 & $1.003-20.12$ & 0.050 & 39.080 & $3.264-467.800$ & 0.004 \\
\hline T stage (T1 vs. T2) & 0.751 & $0.168-3.357$ & 0.71 & & & \\
\hline Risk group (low vs. intermediate) & 0.158 & $0.019-1.311$ & 0.087 & 0.433 & $0.017-11.010$ & 0.61 \\
\hline Neoadjuvant ADT (no vs. yes) & 2.244 & $0.434-11.62$ & 0.34 & & & \\
\hline Number of seeds & 0.963 & $0.907-1.023$ & 0.22 & & & \\
\hline Prostate volume (24 h) & 0.987 & $0.910-1.070$ & 0.92 & & & \\
\hline Prostate D90 (24 h) & 0.934 & $0.886-0.985$ & 0.012 & 1.255 & $0.923-1.706$ & 0.15 \\
\hline ASQ D90 (24 h) & 0.956 & 0.918-0.997 & 0.034 & 0.968 & $0.916-1.023$ & 0.25 \\
\hline PSQ D90 (24 h) & 0.952 & 0.916-0.990 & 0.014 & 0.904 & $0.848-0.964$ & 0.002 \\
\hline AIQ D90 (24 h) & 0.995 & $0.957-1.033$ & 0.78 & & & \\
\hline PIQ D90 (24 h) & 0.973 & $0.937-1.009$ & 0.14 & 0.958 & 0.919-0.999 & 0.048 \\
\hline
\end{tabular}

$H R$ - hazard ratio, Cl - confidence interval, PSA - prostate specific antigen, ADT - androgen deprivation therapy, D90 - minimal dose received by $90 \%$ of volume, $A S Q$ - anterior-superior quadrant, $P S Q$ - posterior-superior quadrant, AIQ - anterior-inferior quadrant, PIQ - posterior-inferior quadrant

segments were different. The segments were divided simply with the midpoint of the whole prostate on reconstructed images, and Spadinger and Sidhu et al. divided the segments with the midpoint of the prostate contour on each axial image [29]. Although the present dividing method has a disadvantage, in that volume deviation of the anterior and posterior regions tends to occur, the present method is simple, intuitive, and easy to apply in various situations, not only in post-implant assessment, but also in real-time planning. In particular, the present method is considered to be more useful with sagittal views, and the results of the present study showing that the information about the craniocaudal direction is important support the utility of this method. Furthermore, 
Table 3B. Univariate and multivariate analyses of prognostic factors using variables obtained from post-implant CT at 1 month

\begin{tabular}{|c|c|c|c|c|c|c|}
\hline \multirow[t]{2}{*}{ Variables } & \multicolumn{3}{|c|}{ Univariate analysis } & \multicolumn{3}{|c|}{ Multivariate analysis } \\
\hline & $\mathrm{HR}$ & $95 \% \mathrm{Cl}$ & $p$-value & $\mathrm{HR}$ & $95 \% \mathrm{Cl}$ & $p$-value \\
\hline Age & 0.977 & $0.881-1.084$ & 0.67 & & & \\
\hline PSA & 1.287 & $1.015-1.633$ & 0.037 & 1.501 & $1.109-2.030$ & 0.008 \\
\hline Gleason score ( $\leq 6$ vs. 7 ) & 4.492 & $1.003-20.12$ & 0.050 & 15.620 & $2.246-108.600$ & 0.005 \\
\hline T stage (T1 vs. T2) & 0.751 & $0.168-3.357$ & 0.71 & & & \\
\hline Risk group (low vs. intermediate) & 0.158 & $0.019-1.311$ & 0.087 & 2.379 & $0.133-42.670$ & 0.56 \\
\hline Neoadjuvant ADT (no vs. yes) & 2.244 & $0.434-11.62$ & 0.34 & & & \\
\hline Number of seeds & 0.963 & $0.907-1.023$ & 0.22 & & & \\
\hline Prostate volume (1 month) & 0.956 & $0.858-1.066$ & 0.42 & & & \\
\hline Prostate D90 (1 month) & 0.953 & $0.916-0.991$ & 0.016 & 0.990 & $0.923-1.061$ & 0.77 \\
\hline ASQ D90 (1 month) & 0.971 & $0.940-1.002$ & 0.067 & 1.012 & $0.961-1.066$ & 0.65 \\
\hline PSQ D90 (1 month) & 0.970 & $0.942-0.999$ & 0.045 & 0.957 & 0.924-0.991 & 0.014 \\
\hline AIQ D90 (1 month) & 1.007 & $0.976-1.038$ & 0.66 & & & \\
\hline PIQ D90 (1 month) & 0.994 & $0.965-1.024$ & 0.71 & & & \\
\hline
\end{tabular}

$H R$ - hazard ratio, Cl - confidence interval, PSA - prostate specific antigen, ADT-androgen deprivation therapy, D90 - minimal dose received by $90 \%$ of volume, $A S Q$ - anterior-superior quadrant, $P S Q$ - posterior-superior quadrant, AIQ - anterior-inferior quadrant, PIQ - posterior-inferior quadrant

we used only standard function of TPS, and it is thought that our method is excellent also in terms of versatility. Differences in patients' characteristics, such as ethnicity and prostate volume, can also be another reason for discrepancy in the results. The posterior region of the prostate contains a major part of the peripheral zone, and pathologically, $70 \%$ of prostate cancers arise in this zone [30]. Thus, the result that delivers an adequate dose to
A

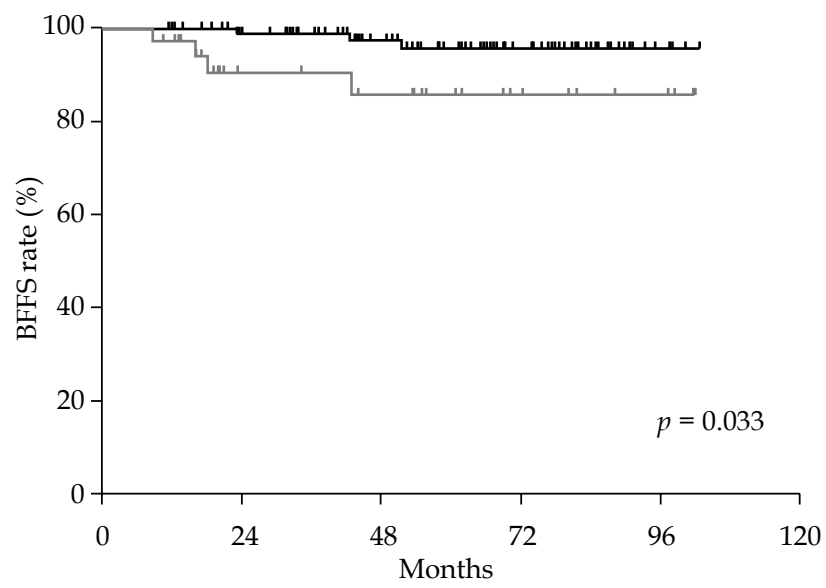

Number at risk

$>145$ Gy

$\leq 145$ Gy

35
B

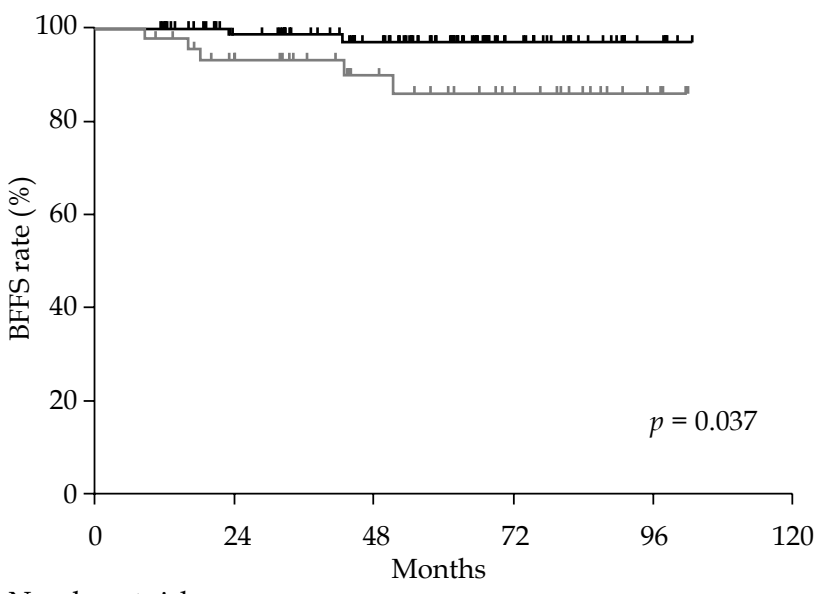

Number at risk

$>160 \mathrm{~Gy}$

85
$\leq 160 \mathrm{~Gy}$

$\begin{array}{ccc}69 & 54 & 35 \\ 37 & 26 & 20 \\ & + \text { PSQ D } 90_{1 \mathrm{~m}}>160 \mathrm{~Gy} \\ -\mathrm{PSQ} & \mathrm{P} 90_{1 \mathrm{~m}} \leq 160 \mathrm{~Gy}\end{array}$

Fig. 2. A) Kaplan-Meier curves of BFFS by $D_{90}$ of PSQ at $24 \mathrm{~h}$ with a cut-off value of $145 \mathrm{~Gy}$. B) Kaplan-Meier curves of BFFS by $\mathrm{D}_{90}$ of PSQ at 1 month with a cut-off value of $160 \mathrm{~Gy}$

BFFS - biochemical failure-free survival, PSQ D90 $24 \mathrm{~h}$ - D90 of PSQ at $24 \mathrm{~h}$, PSQ D90 $0_{1 m}$ - D90 of PSQ at 1 month, D90 - minimal dose received by $90 \%$ of volume, $P S Q$ - posterior-superior quadrant 
A

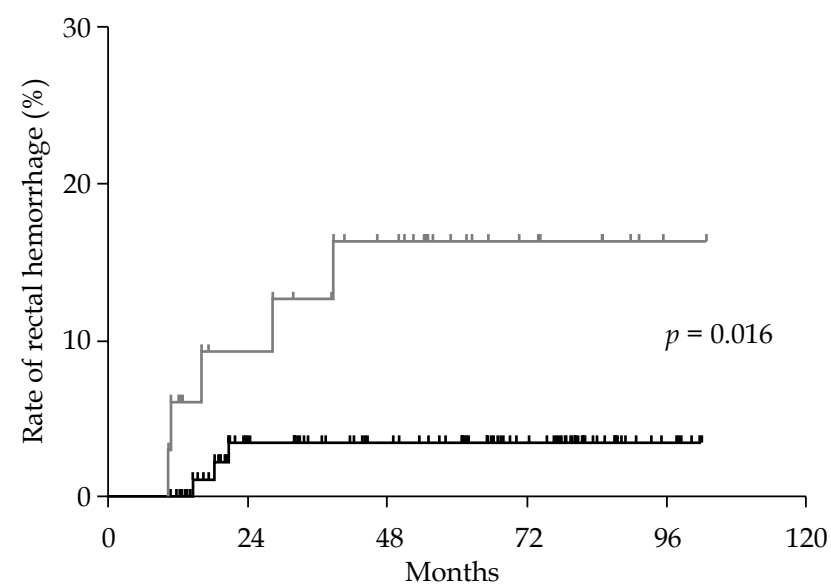

Number at risk

$$
\begin{array}{rrcc}
>195 \mathrm{~Gy} & & \\
33 & 28 & 22 & 9 \\
\leq 195 \mathrm{~Gy} & & & 33 \\
97 & 75 & 57 & 37 \\
& & + \text { PSQ D } 90_{1 \mathrm{~m}}>195 \mathrm{~Gy} \\
& & -\mathrm{PSQ} \text { D } 90_{1 \mathrm{~m}} \leq 195 \mathrm{~Gy}
\end{array}
$$

B

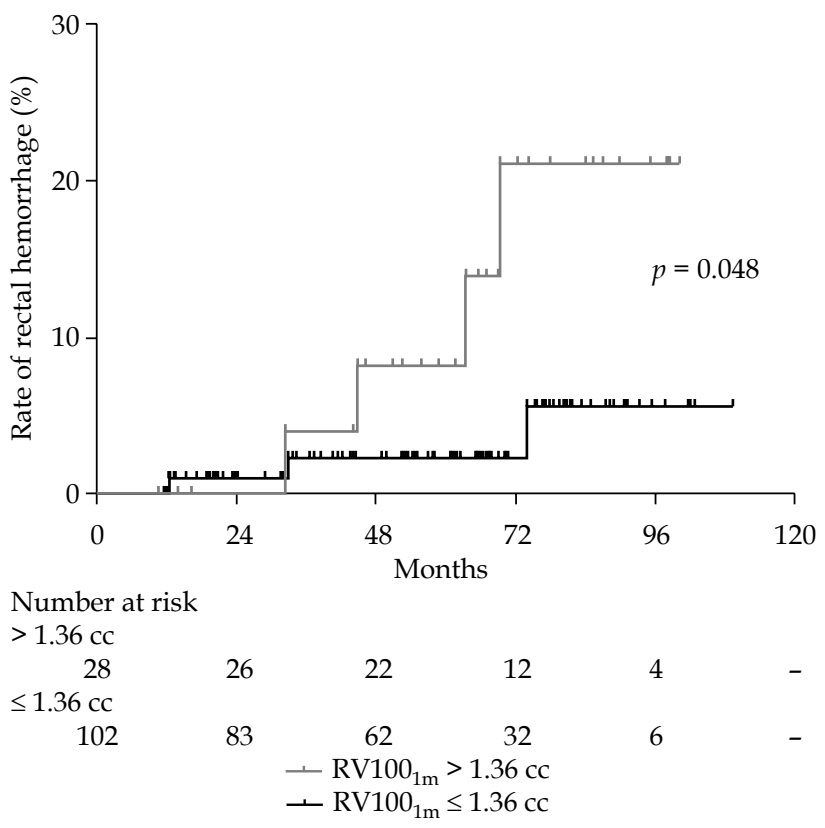

Fig. 3. A) Cumulative Kaplan-Meier curves of the rectal hemorrhage rate by $D_{90}$ of PSQ at 1 month with a cut-off value of $195 \mathrm{~Gy}$. B) Cumulative Kaplan-Meier curves of rectal hemorrhage rate by $V_{100}$ of rectum at 1 month with a cut-off value of $1.36 \mathrm{cc}$ PSQ D90 Im - D90 of PSQ at 1 month, D90 - minimal dose received by 90\% of volume, PSQ -posterior-superior quadrant, RV100 $1 \mathrm{~m}$ - V100 of rectum at 1 month

PSQ is correlated with BFFS, and is meaningful in terms of pathology of prostate cancer.

The present study indicates the utility of post-implant dosimetric analysis not only at 1 month, but also $24 \mathrm{~h}$ after the implant procedure. Though there are many reports about post-implant dosimetry with CT and/or MRI images obtained at 2-6 weeks after the procedure, the number of reports using images at $24 \mathrm{~h}$ is relatively small [16]. To the best of our knowledge, this is the first report dealing with the correlation between BFFS and regional dosimetry of the prostate using images obtained at $24 \mathrm{~h}$ after the procedure. The result that regional dosimetry at $24 \mathrm{~h}$ can predict the BFFS is meaningful, because it facilitates early compensation for a dose deficiency. A prospective study is needed to investigate whether compensating for a dose deficiency can contribute to improving BFFS.

In the present study, the cut-off value of PSQ D90 was $145 \mathrm{~Gy}$ at $24 \mathrm{~h}$ and $160 \mathrm{~Gy}$ at 1 month. Each value was significant for predicting BFFS. In a series of 686 patients, Shiraishi et al. [10] showed that D90 of the whole prostate on day 1 or day 30 was related to biochemical control on multivariate analysis. They also showed that the cut-off values for the day 1 D90 and the day 30 D90 were 163 Gy and 175 Gy, respectively. They indicated that dose escalation is needed to improve the results of LDR brachytherapy. In the present study, adequate dose distribution was shown to be needed for better BFFS, but there was no significant relationship between D90 of the whole prostate and BFFS. Considering the results of the present study, D90 of PSQ may be more useful than D90 of the whole prostate for predicting BFFS in patients with localized prostate cancer.
On multivariate analysis, PSA and Gleason score were significant factors for biochemical failure, while $\mathrm{T}$ stage and risk group were not significant. The following points can be considered to explain the results: (a) many of the patients in this study were classified as T1c or T2a, and there was no significant difference in risk of biochemical failure between the two groups [31]; (b) many of the intermediate-risk patients had a good prognosis, since they were classified into the "favorable intermediate-risk group" [32].

It was found that PSQ D90 $1 \mathrm{~m}$ and V100 of the rectum are significant factors of rectal hemorrhage. It is well known that the incidence rate of rectal hemorrhage can correlate with V100 of the rectum [33,34]. Despite the significant correlation between rectal hemorrhage and "rectal dose", few reports on the relationship between rectal hemorrhage and "(regional) prostatic dose" have been published to date. Furthermore, PSQ is not anatomically closer to rectum than PIQ. One plausible reason is that PSQ is larger than PIQ, and high-dose administration to PSQ contributes more to dose escalation of rectum than PIQ. The cut-off value of PSQ D90 $1 \mathrm{~m}$ for rectal hemorrhage was $195 \mathrm{~Gy}$ on ROC analysis. Although the AUC of 0.69 on ROC analysis can be considered to reflect low accuracy; the log-rank test showed that the cut-off value (PSQ D90 $1 \mathrm{~m}$ : $195 \mathrm{~Gy}$ ) was significant for predicting rectal hemorrhage. Thus, PSQ D90 $1 \mathrm{~m}$ may be a useful factor for predicting rectal toxicity. As mentioned above, adequate dose administration to PSQ of the prostate is needed to improve BFFS, while excessive dose escalation can lead to worsening of late complications.

In the treatment of patients who have a higher risk in PSQ from the results of biopsy or MRI study, higher 
dose administration may be needed to improve BFFS. The prescription dose was $145 \mathrm{~Gy}$ in the present study. In a series of 686 patients, Shiraishi et al. [10] treated with a prescribed dose of $145 \mathrm{~Gy}$ or $160 \mathrm{~Gy}$, and they showed that dose escalation is needed to improve results. Thus, we think that there is a room for dose escalation. In contrast, excessive dose escalation can lead to worsening of late complications. In terms of seed selection, loose seed was used in the present study, and this might have led to seed migration and unintended heterogeneity of dose distribution. Linked seed can reduce the probability of seed migration, and it may decrease excessive dose administration to the rectum or other organs.

In this study, D90 of PIQ at $24 \mathrm{~h}$ was significantly lower than that of other quadrants. The PIQ is a portion close to the rectum, particularly on the caudal side, and the lower dose of PIQ is considered to be a result of prostatic edema or avoiding placing the seeds in the vicinity of the rectum. On the other hand, D90 of PIQ at 1 month was not significantly lower compared with other quadrants. Possible reasons for this result are the proximity of the seeds to the rectum due to improved edema of the prostate or slight migration of the seeds to the caudal side in the prostate.

This study had several limitations. First, this study was limited by its retrospective design and single-center setting, a relatively short follow-up period, and the relatively low numbers of patients and events compared with multi-institutional studies. Although the follow-up period was relatively short, all patients reached PSA nadir after brachytherapy, and this appears sufficient to analyze biochemical control. Second, CT-MRI image fusion technique was performed with MRI images obtained at pre-planning. It is well known that the results of brachytherapy depend greatly on practitioner. Therefore, we used CT-MRI image fusion technique to mitigate variations in contouring related practitioner. The prostatic volume at post-planning differed from that at pre-planning in many patients, and the MRI images were modified to fit the CT images on TPS. Thus, the accuracy of delineation might be less than with MRI images obtained simultaneously. Third, the relationship between BFFS and biopsy information, such as number or percentage of positive cores and localization or volume of prostate cancer, was not evaluated. Fourth, neoadjuvant ADT has been administered to 60 patients $(46.2 \%)$ to reduce the prostate volume, and the influence of ADT could not be completely excluded. Neoadjuvant ADT has been administered to 5 of 7 patients with biochemical recurrence, and neoadjuvant ADT showed no significant effect in BFFS on univariate analysis. Therefore, the influence of neoadjuvant ADT on results of this study is considered to be sufficiently small. Further new prospective investigation is needed to confirm the result of this study, and to assess the correlations of regional dosimetrics, biopsy information, and BFFS.

\section{Conclusions}

Post-implant D90 of PSQ is significantly associated with BFFS for localized prostate cancer not only at 1 month, but also at $24 \mathrm{~h}$ after implant procedure, with cut-off values of $160 \mathrm{~Gy}$ and $145 \mathrm{~Gy}$, respectively. D90 of PSQ at 1 month is also a significant factor for rectal hemorrhage.

\section{Disclosure}

Authors report no conflict of interest.

\section{References}

1. Attard G, Parker C, Eeles RA et al. Prostate cancer. Lancet 2016; 387: 70-82.

2. Spadinger I, Chu J, Afsari Golshan $M$ et al. Regional dose metrics as predictors of biochemical failure and local recurrence after low-dose-rate prostate brachytherapy. Brachytherapy 2015; 14: 350-358.

3. Dinkla AM, Pieters BR, Koedooder K et al. Prostate volume and implant configuration during 48 hours of temporary prostate brachytherapy: limited effect of oedema. Radiat Oncol 2014; 9: 272.

4. Waterman FM, Dicker AP. Probability of late rectal morbidity in $125 \mathrm{I}$ prostate brachytherapy. Int J Radiat Oncol Biol Phys 2003; 55: 342-353.

5. Taussky D, Igidbashian L, Donath D et al. Is intraoperative real-time dosimetry in prostate seed brachytherapy predictive of biochemical outcome? J Contemp Brachytherapy 2017; 9: 304-308.

6. Roach M, 3rd, Hanks G, Thames H, Jr. et al. Defining biochemical failure following radiotherapy with or without hormonal therapy in men with clinically localized prostate cancer: recommendations of the RTOG-ASTRO Phoenix Consensus Conference. Int J Radiat Oncol Biol Phys 2006; 65: 965-974.

7. Stock RG, Stone NN, Tabert A et al. A dose-response study for I-125 prostate implants. Int J Radiat Oncol Biol Phys 1998; 41: 101-108.

8. Nasser NJ, Wang Y, Borg J, Saibishkumar EP. Sector analysis of dosimetry of prostate cancer patients treated with lowdose-rate brachytherapy. Brachytherapy 2014; 13: 369-374.

9. Kirschner AN, Sathiaseelan V, Zhang Y et al. Multisector dosimetry in the immediate post-implant period: significant under dosage of the prostate base. J Contemp Brachytherapy 2014; 6: 33-39.

10. Nath R, Bice WS, Butler WM et al. AAPM recommendations on dose prescription and reporting methods for permanent interstitial brachytherapy for prostate cancer: report of Task Group 137. Med Phys 2009; 36: 5310-5322.

11. Sidhu S, Morris WJ, Spadinger I et al. Prostate brachytherapy postimplant dosimetry: a comparison of prostate quadrants. Int J Radiat Oncol Biol Phys 2002; 52: 544-552.

12. Rodrigues G, Yao X, Loblaw DA et al. Low-dose rate brachytherapy for patients with low- or intermediate-risk prostate cancer: A systematic review. Can Urol Assoc J 2013; 7: 463-470.

13. Martell K, Meyer T, Sia M et al. Parameters predicting for prostate specific antigen response rates at one year post lowdose-rate intraoperative prostate brachytherapy. J Contemp Brachytherapy 2017; 9: 99-105.

14. McNeal JE, Redwine EA, Freiha FS et al. Zonal distribution of prostatic adenocarcinoma. Correlation with histologic pattern and direction of spread. Am J Surg Pathol 1988; 12: 897-906.

15. Peinemann F, Grouven U, Hemkens LG et al. Low-dose rate brachytherapy for men with localized prostate cancer. Cochrane Database Syst Rev 2011: Cd008871.

16. Stock RG, Stone NN. Importance of post-implant dosimetry in permanent prostate brachytherapy. Eur Urol 2002; 41: 434-439. 
17. Shiraishi Y, Yorozu A, Ohashi T et al. A dose-response analysis of biochemical control outcomes after (125) I monotherapy for patients with favorable-risk prostate cancer. Int J Radiat Oncol Biol Phys 2014; 90: 1069-1075.

18. Bice WS, Jr., Prestidge BR, Sarosdy MF. Sector analysis of prostate implants. Med Phys 2001; 28: 2561-2567.

19. Ishiyama H, Sekiguchi A, Satoh T et al. Dosimetry of permanent interstitial prostate brachytherapy for an interoperative procedure, using O-arm based CT and TRUS. J Contemp Brachytherapy 2016; 8: 7-16.

20. Spadinger I, Morris WJ, Keyes M et al. Quadrant dosimetry as a predictor of biochemical relapse in I-125 prostate brachytherapy. Brachytherapy 2011; 10: 87-97.

21. Zaorsky NG, Davis BJ, Nguyen PL et al. The evolution of brachytherapy for prostate cancer. Nat Rev Urol 2017; 14: 415-439.

22. Yu Y, Anderson LL, Li Z et al. Permanent prostate seed implant brachytherapy: report of the American Association of Physicists in Medicine Task Group No. 64. Med Phys 1999; 26: 2054-2076.

23. Snyder KM, Stock RG, Hong SM et al. Defining the risk of developing grade 2 proctitis following 125I prostate brachytherapy using a rectal dose-volume histogram analysis. Int J Radiat Oncol Biol Phys 2001; 50: 335-341.

24. Nag S, Bice W, DeWyngaert $K$ et al. The American Brachytherapy Society recommendations for permanent prostate brachytherapy postimplant dosimetric analysis. Int J Radiat Oncol Biol Phys 2000; 46: 221-230.

25. Papagikos MA, Deguzman AF, Rossi PJ et al. Dosimetric quantifiers for low-dose-rate prostate brachytherapy: is V(100) superior to D(90)? Brachytherapy 2005; 4: 252-258.

26. Serrano NA, Anscher MS. Favorable vs Unfavorable Intermediate-Risk Prostate Cancer: A Review of the New Classification System and Its Impact on Treatment Recommendations. Oncology 2016; 30: 229-236.

27. Morris WJ, Keyes M, Spadinger I et al. Population-based 10year oncologic outcomes after low-dose-rate brachytherapy for low-risk and intermediate-risk prostate cancer. Cancer 2013; 119: 1537-1546.

28. Nag S, Beyer D, Friedland J et al. American Brachytherapy Society (ABS) recommendations for transperineal permanent brachytherapy of prostate cancer. Int J Radiat Oncol Biol Phys 1999; 44: 789-799.

29. Merrick GS, Butler WM, Dorsey AT et al. Influence of timing on the dosimetric analysis of transperineal ultrasound-guided, prostatic conformal brachytherapy. Radiat Oncol Investig 1998; 6: 182-190.

30. Potters L, Cao Y, Calugaru E et al. A comprehensive review of CT-based dosimetry parameters and biochemical control in patients treated with permanent prostate brachytherapy. Int J Radiat Oncol Biol Phys 2001; 50: 605-614.

31. Butler WM, Stewart RR, Merrick GS. A detailed radiobiological and dosimetric analysis of biochemical outcomes in a case-control study of permanent prostate brachytherapy patients. Med Phys 2009; 36: 776-787.

32. Merrick GS, Butler WM, Grimm P et al. Multisector prostate dosimetric quality: analysis of a large community database. Brachytherapy 2014; 13: 146-151.

33. Willins J, Wallner K. Time-dependent changes in CT-based dosimetry of I-125 prostate brachytherapy. Radiat Oncol Investig 1998; 6: 157-160.

34. Mortier P, Bastide C, Lechevallier E et al. Oncological results of active surveillance in prostate cancer: A retrospective multicentric cohort. Prog Urol 2017; 27: 38-45. 